T. Matsuzawa

Nagoya Math. J.

Vol. 52 (1973), 61-84

\title{
ON SOME DEGENERATE PARABOLIC EQUATIONS II
}

\author{
TADATO MATSUZAWA
}

§ 1. Introduction. In the article I: [8], we have proved the hypoellipticity of a degenerate parabolic equation of the form:

$$
P u=\frac{\partial u}{\partial t}-a(x, t) \frac{\partial^{2} u}{\partial x^{2}}+b(x, t) \frac{\partial u}{\partial x}+c(x, t) u=f,
$$

where the coefficients $a(x, t), b(x, t)$ and $c(x, t)$ are complex valued smooth functions. The fundamental assumption on the coefficients is that $\mathscr{R}_{e} a(x, t)$ satisfies the condition of Nirenberg and Treves ([8], (1.5)). To prove the hypoellipticity we have constructed recurcively the parametrices as pseudodifferential operators with parameter. This method may be viewed as an improvement of that of [9] and [7]. We have analyzed the properties of these parametrices by estimating the symbols with parameter associated with the given operator. We shall summerize these results in $\S 3$.

The aim of this paper is to solve the Cauchy problem for an equation of the type (1.1). To this end, we shall apply the Levi method, which is described in detail in the book [2], Ch. 9.

The first step is to construct the fundamental solution starting with the above mentioned parametrices $(\S 4, \S 5)$. There we need to obtain the precise estimates of the singularity of parametrices. Those estimates are derived by observing the order of the corresponding symbols more precisely than [8]. The difficulty arises in constructing the fundamental solution since the singularity of the derivative of the first parametrix can no longer be integrable. We shall be able to obtain the fundamental solution as a solution of an integral equation by using the $\mu$-th parametrix with $\mu$ sufficiently large (§5). As soon as the fundamental solution is obtained, we can prove the existence of the solution of the Cauchy problem by forming the integral expression ( $(6)$.

Received May 28, 1973. 
The second step is to prove the uniqueness of the solution. The estimates of the derivatives of the fundamental solution are not available yet, so, in $\S 7$, we shall make use of the method of [1] to prove the uniqueness of the solution, where it is necessary to assume all the coefficients of $P$ are real valued. We shall therefore assume that the coefficients $a, b, c$ are real valued smooth functions throughout this paper, while such an assumption is not necessary in the first step, namely in constructing the fundamental solution. The precise condition and the formulation of the Cauchy problem will be prescribed in $\S 2$. These two steps will prove Theorem 2.1 which is our main result.

Finally, we remark that the degenerate Cauchy problem for a wide class of equations has been treated in [11], [12] by the method of elliptic regularization, and in [6] by using the method of semi-group theory. Our approach is based on the application of the theory of pseudodifferential operators for a classical treatment of the parabolic equations.

\$. Main results. Let $a(x, t), b(x, t)$ and $c(x, t)$ be real valued infinitely differentiable functions defined in $R \times I, I=[0, \mathrm{~T}], T>0$, and satisfy the following conditions:

For any integer $m \geqq 0,\left|D_{x}^{m} a(x, t)\right|,\left|D_{x}^{m} b(x, t)\right|$ and $\left|D_{x}^{m} c(x, t)\right|$ are bounded in $R \times I$ where $D_{x}^{m}=\frac{\partial^{m}}{\partial x^{m}}$;

$$
a(x, t) \geqq 0 \quad \text { in } R \times I ;
$$

there exist an integer $\sigma \geqq 1$ and a real number $\delta>0$ such that

$$
\int_{t^{\prime}}^{t} a(x, \tau) d \tau \geqq \delta\left(t-t^{\prime}\right)^{\sigma} \quad x \in R, \quad 0 \leqq t^{\prime} \leqq t \leqq T ;
$$

$$
\begin{aligned}
& \qquad\left|a_{x}(x, t)\right| \leqq C a(x, t)^{1 / 2} \quad \text { in } R \times I ; \\
& |b(x, t)| \leqq C a(x, t)^{1 / 2} \quad \text { in } R \times I, \text { where } C \text { denotes a positive } \\
& \text { constant }
\end{aligned}
$$

Under these conditions, we shall solve the following Cauchy problem:

$$
\begin{array}{cc}
P u=\frac{\partial u}{\partial t}-a(x, t) \frac{\partial^{2} u}{\partial x^{2}}+b(x, t) \frac{\partial u}{\partial t}+c(x, t) u= & f(x, t) \\
u(x, 0)=\varphi(x) \quad \text { on } R, & \text { in } R \times(0, T),
\end{array}
$$


where $f(x, t)$ is a measurable function in $R \times I$ and $\varphi(x)$ is a continuous function in $R$.

By a solution of (2.6), (2.7) we mean a function $u=u(x, t)$ in $C(R \times I)$ which satisfies the equation (2.6) in the distribution sense in $R \times(0, T)$ and satisfies the initial condition (2.7) in the usual sense. Our main result is the following.

THEOREM 2.1. Let $f(x, t)$ be a measurable function in $R \times I$ and $\varphi(x)$ be a continuous function in $R$ and assume that

$$
|f(x, t)| \leqq M \exp \left[k|x|^{2}\right] \quad \text { a.e. in } R \times I \text {, }
$$$$
|\varphi(x)| \leqq M \exp \left[k|x|^{2}\right] \quad \text { in } R
$$

for some positive constants $M$ and $k$. Then there exists a solution $u(x, t)$ of the Cauchy problem (2.6), (2.7) in the strip $R \times\left[0 \leqq t \leqq t_{0}\right]$, where $t_{0}\left(0<t_{0} \leqq T\right)$ is a constant depending on the operator $P$ and where

$$
|u(x, t)| \leqq \text { const. } \exp \left[k^{\prime}|x|^{2}\right] \quad \text { in } R \times\left[0 \leqq t \leqq t_{0}\right]
$$

for some constant $k^{\prime}$. The solution is unique in the class of continuous functions satisfying (2.10) with some constant $k^{\prime}$. Furthermore the solution $u(x, t)$ is in $C^{\infty}\left(R \times\left[0<t \leqq t_{0}\right]\right)$ if $f(x, t)$ is in $C^{\infty}(R \times I)$.

In $\S 6$, we shall prove the existence part of this theorem, and in $\S 7$ we shall prove the uniqueness of the solution. We remark that the solution exists in the whole strip $R \times[0, T]$ if we replace the condition (2.8), (2.9) by

$$
\begin{aligned}
|f(x, t)| & \leqq M \exp \left[k|x|^{2-s}\right] & & \text { a.e. in } R \times I, \\
|\varphi(x)| & \leqq M \exp \left[k|x|^{2-s}\right] & & \text { in } R, \quad \varepsilon>0 .
\end{aligned}
$$

EXAMPLE: The coefficients of the operator

$$
P_{k}=\frac{\partial}{\partial t}-\left(t^{k}+e^{-1 /|x|}\right) \frac{\partial^{2}}{\partial x^{2}}+t^{[(k+1) / 2]} \frac{\partial}{\partial x}+\text { const., } k \text { integer }>0
$$

satisfy the conditions $(2.1) \sim(2.5)$ in $R_{x} \times[0, \mathrm{~T}](\mathrm{T}>0)$ with $\sigma=k+1$.

\section{§3. Application of the results of the article I: [8].}

We can construct the parametrices for the operator $P$ as in [8] under the conditions $(2.1) \sim(2.5)$. We note that we have treated the case where $\sigma=2 \ell+1, \ell \geqq 0$ and the coefficients $a, b, c$ have been assumed to be 
complex valued functions (in [8]). We recall the procedure in the following. We set

$$
\begin{aligned}
& L_{1}=\frac{\partial}{\partial t}+a(x, t) \xi^{2}, \\
& L_{2}=-i \xi a(x, t) \frac{\partial}{\partial x}-a(x, t) \frac{\partial^{2}}{\partial x^{2}}+b(x, t)\left(\frac{\partial}{\partial x}+i \xi\right)+c(x, t),
\end{aligned}
$$

for $(x, t, \xi) \in R_{x} \times I \times R_{\xi}$. Consider the problem:

$$
\begin{gathered}
L_{1} K_{0}=\left(\frac{\partial}{\partial t}+a(x, t) \xi^{2}\right) K_{0}\left(x, \xi ; t, t^{\prime}\right)=0 \quad \text { in } R_{x} \times R_{\xi} \times \Delta, \\
\Delta \equiv\left\{\left(t, t^{\prime}\right) ; 0 \leqq t^{\prime}<t \leqq T\right\} \\
\left.K_{0}\left(x, \xi ; t, t^{\prime}\right)\right|_{t=t^{\prime}}=1, \\
K_{0}\left(x, \xi ; t, t^{\prime}\right)=0 \quad \text { if } 0 \leqq t<t^{\prime} \leqq T .
\end{gathered}
$$

Then we have the solution

$$
K_{0}\left(x, \xi ; t^{\prime}\right)=\left\{\begin{array}{c}
\exp \left[-\int_{t^{\prime}}^{t} a(x, \tau) d \tau \cdot \xi^{2}\right], \quad\left(x, \xi, t, t^{\prime}\right) \in R \times R_{\xi} \times \bar{\Delta}, \\
0 \quad \text { when } 0 \leqq t<t^{\prime} \leqq T
\end{array}\right.
$$

We note that the notion of the set $\Delta$ is slightly different from that of [8]. For $j=0,1,2, \cdots$ we define recurcively the symbol functions $K_{j}\left(x, \xi ; t, t^{\prime}\right)$ as the solution of the problem:

$$
\begin{gathered}
L_{1} K_{j+1}\left(x, \xi ; t, t^{\prime}\right)=-L_{2} K_{j}\left(x, \xi ; t, t^{\prime}\right) \quad \text { in } R_{x} \times R_{\xi} \times \Delta, \\
\left.K_{j+1}\left(x, \xi ; t, t^{\prime}\right)\right|_{t=t^{\prime}}=0, \\
K_{j+1}\left(x, \xi ; t, t^{\prime}\right)=0 \quad \text { if } 0 \leqq t<t^{\prime} \leqq T .
\end{gathered}
$$

The $K_{j}$ 's satisfy.

$$
K_{j+1}\left(x, \xi ; t, t^{\prime}\right)=\left\{\begin{array}{cc}
-\int_{t^{\prime}}^{t} K_{0}(x, \xi ; t, s) L_{2} K_{j}\left(x, \xi ; s, t^{\prime}\right) d s, & \\
& \left(x, \xi, t, t^{\prime}\right) \in R_{x} \times R_{\xi} \times \bar{\Delta} \\
0, & \text { when } 0 \leqq t<t^{\prime} \leqq T .
\end{array}\right.
$$

As in [8], we have the following proposition:

Proposition 3.1. (cf. [8], Prop. 4.1, Prop. 5.1) For any $\varepsilon>0$ and for any integer $\alpha$ and $\beta \geqq 0$, we have 
(3.9)

$$
\begin{aligned}
& K_{j}\left(x, \xi ; t, t^{\prime}\right) \in \mathscr{E}\left(\Delta ; S^{-\infty}\left(R_{x} \times R_{\xi}\right)\right) \cap \bigcap_{p \geqq 0} \mathscr{E}^{p}\left(\bar{\Delta} ; S_{1,(\sigma-1) / \sigma}^{\varepsilon+2 p-j / \sigma}\left(R_{x} \times R_{\xi}\right)\right), \\
& j=0,1,2, \cdots, \\
& \text { (3.10) } \quad \mid\left(K_{0}\left(x, \xi ; t, t^{\prime}\right)-1\right)(1+|\xi|)^{-\varepsilon} \rightrightarrows 0 \text { in } R_{x} \times R_{\xi} \quad \text { as } t \downarrow t^{\prime} \text {, } \\
& \left|\left(D_{t, t^{\prime}}^{p} D_{x}^{\beta} D_{\xi}^{\alpha} K_{j}\right)(1+|\xi|)^{(-\beta(\sigma-1)) / \sigma-2 p+\alpha+j / \sigma-\varepsilon}\right| \rightrightarrows 0 \text { in } \\
& R_{x} \times R_{\xi} \quad \text { as } t \downarrow t^{\prime} \quad \text { for } 0 \leqq p<j .
\end{aligned}
$$

For the notations in the proposition we refer to the article [8].

By virtue of Proposition 3.1, for every $j \geqq 0$, we can define a distribution $\mathscr{K}_{j}=\mathscr{K}_{j}\left(x, y, t, t^{\prime}\right) \in \mathscr{D}^{\prime}\left(R_{x} \times R_{y} \times I_{t} \times I_{t^{\prime}}\right)$ by an oscillatory integral :

$$
\mathscr{K}_{j}\left(x, y, t, t^{\prime}\right)=(2 \pi)^{-1 / 2} \int_{-\infty}^{\infty} e^{i(x-y) \xi} K_{j}\left(x, \xi ; t, t^{\prime}\right) d \xi
$$

As in [8], we can show that

(i ) $\left.P_{x, t}\left[\mathscr{K}_{0}+\cdots+\mathscr{K}_{\mu}\right]=\delta(x-y), t-t^{\prime}\right)+(2 \pi)^{-1 / 2} \int_{-\infty}^{\infty} e_{i}^{(x-y) \xi} L_{2} K_{\mu}$ $\left(x, \xi ; t, t^{\prime}\right) d \xi \quad$ in $R_{x} \times R_{y} \times I_{t} \times I_{t^{\prime}}$,

(ii) $\quad \mathscr{K}_{j}\left(x, y, t, t^{\prime}\right) \in C^{\infty}(W), \quad j=0,1,2, \cdots$, $W=\left\{\left(x, y, t, t^{\prime}\right) \in R_{x} \times R_{y} \times I \times I ;|x-y|+\left|t-t^{\prime}\right|>0\right\}$

(iii) $\mathscr{K}_{j}\left(x, y, t, t^{\prime}\right)$ is very regular in the sense of Schwartz [15], $j=0,1,2, \cdots$, that is to mean the mappings

$$
\begin{aligned}
\psi\left(y, t^{\prime}\right) & \mapsto \iint_{R \times I} \mathscr{K}_{j}\left(x, y, t, t^{\prime}\right) \psi\left(y, t^{\prime}\right) d y d t^{\prime}, \\
\varphi(x, t) & \mapsto \iint_{R \times I} \mathscr{K}_{j}\left(x, y, t, t^{\prime}\right) \varphi(x, t) d x d t
\end{aligned}
$$

define linear continuous transformations from $\mathscr{D}(R \times I)$ into $\mathscr{E}(R \times I)$. These are extended to the linear continuous transformation from $\mathscr{E}^{\prime}(R \times I)$ into $\mathscr{D}^{\prime}(R \times I)$ by continuity (cf. [8], Prop. 4.2).

(iv) the second term in the right of (i) is also very regular and becomes smoother in $R_{x} \times R_{y} \times I \times I$ according as $\mu$ becomes larger.

From these facts we obtain the following theorem. 
THEOREM 3.1. (cf. [8], Theorem 1.1). The operator $P$ is hypoelliptic in the strip $R \times I$.

§4. Precise estimates of $D_{x}^{m} \mathscr{K}_{j}\left(x, y, t, t^{\prime}\right), m, j=0,1,2, \cdots$

We set

$$
\begin{aligned}
A=A\left(x, t, t^{\prime}\right)=\int_{t^{\prime}}^{t} a(x, \tau) d \tau \quad\left(x, t, t^{\prime}\right) \in R \times \bar{\Delta}, \\
\Delta=\left\{\left(t, t^{\prime}\right) \mid 0 \leqq t^{\prime}<t \leqq T\right\} .
\end{aligned}
$$

Then by the assumptions $(2.1) \sim(2.4)$ we have

$$
\begin{gathered}
\delta\left(t-t^{\prime}\right)^{\sigma} \leqq A\left(x, t, t^{\prime}\right) \leqq C\left(t-t^{\prime}\right) \quad\left(x, t, t^{\prime}\right) \in R \times \bar{\Delta} \\
\left|\int_{t^{\prime}}^{t} a_{x}(x, \tau) d \tau\right| \leqq C_{1}\left(t-t^{\prime}\right)^{1 / 2} A^{1 / 2} \leqq C_{2}\left(t-t^{\prime}\right) \quad\left(x, t, t^{\prime}\right) \in R \times \bar{\Delta} .
\end{gathered}
$$

In the following we use the symbols $C, C_{1}, C_{2}, \ldots$ to express the different positive constants.

Now we recall that

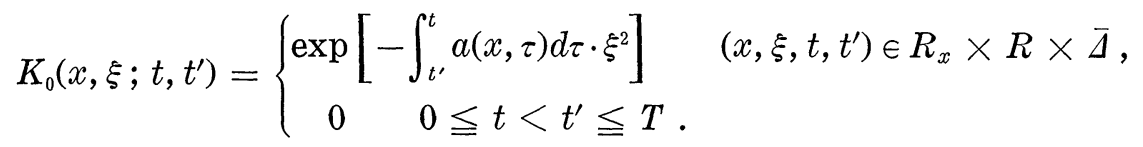

Substituting $\xi$ by $\zeta=\xi+i \eta \in C$, we can extend the domain of $K_{0}$ to the set $R_{x} \times C \times I \times I$ :

$$
K_{0}\left(x, \zeta ; t, t^{\prime}\right)=\left\{\begin{array}{c}
\exp \left[\int_{t^{\prime}}^{t} a(x, \tau) d \tau \cdot(\xi+i \eta)^{2}\right], \quad\left(x, \zeta, t, t^{\prime}\right) \in R \times C \times \bar{\Delta} \\
0 \quad 0 \leqq t<t^{\prime} \leqq T
\end{array}\right.
$$

Lemma 4.1. ((cf. [8], Prop. 4.1) For every integer $m \geqq 0$ we have

$$
\begin{array}{r}
\left|D_{x}^{m} K_{0}\left(x, \zeta ; t, t^{\prime}\right)\right| \leqq C_{m}(1+|\xi|+|\eta|)^{(m(\sigma-1)) / \sigma} \exp \left[-\delta_{1} A \xi^{2}+\delta_{2} A \eta^{2}\right], \\
\left(x, \zeta, t, t^{\prime}\right) \in R \times C \times \bar{\Delta},
\end{array}
$$

where $C_{m}, \delta_{1}, \delta_{2}$ are positive constants depending only on $m$.

Proof. For $m=0$, we easily have

$$
\left|K_{0}\left(x, \zeta ; t, t^{\prime}\right)\right|=\exp \left[-A \xi^{2}+A \eta^{2}\right], \quad\left(t, t^{\prime}\right) \in \bar{\Delta} .
$$

For a positive integer $m, D_{x}^{m} K_{0}\left(x, \zeta ; t, t^{\prime}\right)$ is expressed as a linear combination of terms 


$$
\begin{aligned}
{\left[\prod_{j}\left(\int_{t^{\prime}}^{t} D_{x}^{m^{(i)}} a(x, \tau) d \tau(\xi+i \eta)^{2}\right)\right] } & \exp \left[-\int_{t^{\prime}}^{t} a(x, \tau) d \tau(\xi+i \eta)^{2}\right] \\
0 & <m^{(j)}, m^{(l)}+\cdots+m^{(j)}+\cdots=m .
\end{aligned}
$$

We are now ready to analyse each factor in (4.5). For a factor with $m^{(j)}=1$ we see by $(4.2)$

$$
\left|\int_{t^{\prime}}^{t} a_{x}(x, \tau) d \tau(\xi+i \eta)^{2}\right| \leqq C\left(t-t^{\prime}\right)^{1 / 2} A^{1 / 2}\left(\xi^{2}+\eta^{2}\right), \quad\left(t, t^{\prime}\right) \in \bar{\Delta}
$$

Then by (4.1) we have

$$
\begin{aligned}
& \left(t-t^{\prime}\right)^{1 / 2} A^{1 / 2} \xi^{2} \exp \left[-\delta_{1} A \xi^{2}\right] \\
& \quad \leqq|\xi|^{(\sigma-1) / \sigma}\left(\left(t-t^{\prime}\right)^{\sigma} \xi^{2}\right)^{1 / 2 \sigma}\left(A \xi^{2}\right)^{1 / 2} \exp \left[-\delta_{1} A \xi^{2}\right] \\
& \quad \leqq C_{\varepsilon}|\xi|^{(\sigma-1) / \sigma} \exp \left[-\left(\delta_{1}-\varepsilon\right) A \xi^{2}\right],
\end{aligned}
$$

for any $\varepsilon$ such that $0<\varepsilon<\delta_{1}$. Similarly we have

$$
\left(t-t^{\prime}\right)^{1 / 2} A^{1 / 2} \eta^{2} \exp \left[\delta_{2} A \eta^{2}\right]=C_{1}|\eta|^{(\sigma-1) / \sigma} \exp \left[\left(\delta_{2}+C_{2}\right) A \eta^{2}\right]
$$

with some positive constants $C_{1}$ and $C_{2}$. Next, for a factor with $m^{(j)} \geqq 2$ we have

$$
\begin{aligned}
\left|\int_{t^{\prime}}^{t} D_{x}^{m(j)} a(x, \tau) d \tau(\xi+i \eta)^{2}\right| & \leqq C\left(t-t^{\prime}\right)\left(\xi^{2}+\eta^{2}\right) \\
& \leqq C\left(|\xi|^{(2(\sigma-1)) / \sigma}+|\eta|^{(2(\sigma-1)) / \sigma}\right)\left(\left(t-t^{\prime}\right)^{\sigma}\left(\xi^{2}+\eta^{2}\right)\right)^{1 / \sigma}
\end{aligned}
$$

Hence we have, as above, for $m^{(j)} \geqq 2$

$$
\begin{aligned}
& \left|\int_{t^{\prime}}^{t} D_{x}^{m(j)} a(x, \tau) d \tau(\xi+i \eta)^{2}\right| \cdot \exp \left[-\delta_{1} A \xi^{2}+\delta_{2} A \eta^{2}\right] \\
& \quad \leqq C_{1}\left(|\xi|^{(2(\sigma-1)) / \rho}+|\eta|^{(2(\sigma-1)) / \sigma}\right) \exp \left[-\left(\delta_{1}-\varepsilon\right) A \xi^{2}+\left(\delta_{2}+C_{2}\right) A \eta^{2}\right], \\
& \quad 0<\varepsilon<\delta_{1} .
\end{aligned}
$$

The assumption (2.1) has been assumed throughout the proof of Lemma 4.1, while we have used the same symbols $C_{1}, C_{2}, \delta_{1}, \delta_{2}, \ldots$ although they differ in different cases. Combining (4.6), (4.7) and (4.8) we prove Lemma 4.1.

Now we recall that

$$
K_{j+1}\left(x, \xi ; t, t^{\prime}\right)=\left\{\begin{array}{c}
-\int_{t^{\prime}}^{t} K_{0}(x, \xi ; t, s) L_{2} K_{j}\left(x, \xi ; s, t^{\prime}\right) d s, \quad\left(t, t^{\prime}\right) \in \bar{\Delta} \\
0 \quad 0 \leqq t^{\prime}<t \leqq T
\end{array}\right.
$$

where 


$$
L_{2}=-i \xi a(x, t) \frac{\partial}{\partial x}-a(x, t) \frac{\partial^{2}}{\partial x^{2}}+b(x, t)\left(\frac{\partial}{\partial x}+i \xi\right)+c(x, t)
$$

We can also extend the domain of $K_{j+1}\left(x, \xi ; t, t^{\prime}\right)$ by substituting $\xi$ be $\zeta=\xi+i \eta$.

Lemma 4.2. (cf. [8], Prop. 5.1) We have

$$
\begin{array}{r}
\left|D_{x}^{m} K_{j}\left(x, \zeta ; t, t^{\prime}\right)\right| \leqq C_{m, j}(1+|\xi|+|\eta|)^{(m(\sigma-1)) / \sigma-j / \sigma} \exp \left[-\delta_{1} A \xi^{2}+\delta_{2} A \eta^{2}\right], \\
\left(x, \zeta, t, t^{\prime}\right) \in R \times C \times \bar{\Delta}, \quad j, m=0,1,2, \cdots,
\end{array}
$$

where the constants $C_{m, j}, \delta_{1}$ and $\delta_{2}$ depend only on $m$ and $j$.

Proof. We shall use mathematical induction in $j$. By Lemma 4.1 we have the result in the case $j=0$. Assume the inequality (4.9) for some $j \geqq 0$ to estimate $D_{x}^{m} K_{j+1}$ for $m=0,1,2, \cdots$ :

$$
\begin{aligned}
D_{x}^{m} K_{j+1}\left(x, \zeta ; t, t^{\prime}\right)= & -D_{x}^{m} \int_{t^{\prime}}^{t} K_{0}(x, \zeta ; t, s) L_{2} K_{j}\left(x, \zeta ; s, t^{\prime}\right) d s \\
= & -\sum_{\alpha=0}^{m}\left(\begin{array}{c}
m \\
\alpha
\end{array}\right) \\
& \cdot \int_{t^{\prime}}^{t} D_{x}^{m-\alpha} K_{0}(x, \zeta ; t, s) D_{x}^{\alpha}\left(L_{2} K_{j}\left(x, \zeta ; s, t^{\prime}\right)\right) d s .
\end{aligned}
$$

It suffices to estimate some typical terms in the last expression, and other terms will be treated similarly:

(i) By Lemma 4.1 and by the assumption on $K_{j}$ we have

$$
\begin{aligned}
& I_{m, \alpha}^{(1)} \equiv \mid \int_{t^{\prime}}^{t} D_{x}^{m-\alpha} K_{0}(x, \zeta ; t, s) D_{x}^{\alpha}\left(i \zeta a(x, s) D_{x} K_{j}\left(x, \zeta ; s, t^{\prime}\right) d s \mid\right. \\
& \leqq \sum_{\beta=0}^{\alpha}\left(\begin{array}{c}
\alpha \\
\beta
\end{array}\right) \int_{t^{\prime}}^{t}\left|D_{x}^{m-\alpha} K_{0}(x, \zeta ; t, s) D_{x}^{\alpha-\beta+1} K_{j}\left(x, \zeta ; s, t^{\prime}\right) D_{x}^{\beta}(x, s)\right| d s(|\xi|+|\eta|) \\
& \leqq C \sum_{\beta=0}^{\alpha}(1+|\xi|+|\eta|)^{(m-\beta+1)(\sigma-1) / \sigma-j / \sigma} \exp \left[-\delta_{1} A \xi^{2}+\delta_{2} A \eta^{2}\right] \\
& \cdot \int_{t^{\prime}}^{t}\left|D_{x}^{\beta} a(x, s)\right| d s(|\xi|+|\eta|) .
\end{aligned}
$$

As in the proof of Lemma 4.1 we will estimate each term in the last summation. For the term with $\beta=0$ we have

$$
(1+|\xi|+|\eta|)^{(m+1)(\sigma 1) / \sigma-j / \sigma} \exp \left[-\delta_{1} A \xi^{2}+\delta_{2} A \eta^{2}\right] \cdot \int_{t^{\prime}}^{t} a(x, \tau) d \tau(|\xi|+|\eta|)
$$




$$
\begin{gathered}
\leqq C_{1}(1+|\xi|+|\eta|)^{(m+1)(\sigma-1) / \sigma-j / \sigma-1} \exp \left[-\left(\delta_{1}-\varepsilon\right) A \xi^{2}+\left(\delta_{2}+C_{2}\right) A \eta^{2}\right] \\
\quad\left(0<\varepsilon<\delta_{1}\right) \\
=C_{1}(1+|\xi|+|\eta|)^{(m(\sigma-1) / \sigma-(j+1) / \sigma} \exp \left[-\left(\delta_{1}-\varepsilon\right) A \xi^{2}+\left(\delta_{2}+C_{2}\right) A \eta^{2}\right] .
\end{gathered}
$$

For the term with $\beta=1$, applying (4.2) we have

$$
\begin{gathered}
(1+|\xi|+|\eta|)^{\langle m(\sigma-1)) / \sigma-j / \sigma} \exp \left[-\delta_{1} A \xi^{2}+\delta_{2} A \eta^{2}\right] \int_{t^{\prime}}^{t}\left|a_{x}(x, \tau)\right| d \tau(|\xi|+|\eta|) \\
\leqq C_{1}(1+|\xi|+|\eta|)^{(m(\sigma-1))-(j+1) / \sigma} \exp \left[-\left(\delta_{1}{ }^{-} \varepsilon\right) A \xi^{2}+\left(\delta_{2}+C_{2}\right) A \eta^{2}\right]
\end{gathered}
$$

We use (4.8), then the terms with $\beta \geqq 2$ are bounded by

$$
\begin{gathered}
(1+|\xi|+|\eta|)^{(m-\beta+1)(\sigma-1) / \sigma-j / \sigma+(2(\sigma-1)) / \sigma} \exp \left[-\left(\delta_{1}-\varepsilon\right) A \xi^{2}+\left(\delta_{2}+C_{2}\right) A \eta^{2}\right] \\
\leqq(1+|\xi|+|\eta|)^{(m(\sigma-1)) / \sigma-(j+1) / \sigma} \exp \left[-\left(\delta_{1}-\varepsilon\right) A^{2}+\left(\delta_{2}+C_{2}\right) A \eta^{2}\right]
\end{gathered}
$$

Thus we have

$$
I_{m, \alpha}^{(1)} \leqq C(1+|\xi|+|\eta|)^{(m(\sigma-1)) / \sigma-(j+1) / \sigma} \exp \left[-\delta_{1} A \eta^{2}+\delta_{2} A \eta^{2}\right]
$$

with some constants $C, \delta_{1}$ and $\delta_{2}$ depending only on $m, j$.

(ii) We have

$$
\begin{aligned}
& I_{m, \alpha}^{(2)} \equiv \mid \int_{t^{\prime}}^{t} D_{x}^{m-\alpha} K_{0}(x, \zeta ; t, s) D_{x}^{\alpha}\left(i \zeta b(x, s) K_{j}\left(x, \zeta ; s, t^{\prime}\right) d s \mid\right. \\
& \leqq \sum_{\beta=0}^{\alpha}\left(\begin{array}{c}
\alpha \\
\beta
\end{array}\right) \int_{t^{\prime}}^{t}\left|D_{x}^{m-\alpha} K_{0}(x, \zeta ; t, s) D_{x}^{\alpha-\beta} K_{j}\left(x, \zeta ; s, t^{\prime}\right) D_{x}^{\beta} b(x, s)\right| d s(|\xi|+|\eta|) \\
& \leqq C \sum_{\beta=0}^{\alpha}(1+|\xi|+|\eta|)^{(m-\beta)(\sigma-1) / \sigma-j / \sigma} \cdot \exp \left[-\delta_{1} A \xi^{2}+\delta_{2} A \eta^{2}\right] \\
& \cdot \int_{t^{\prime}}^{t}\left|D_{x}^{\beta} b(x, s)\right| d s(|\xi|+|\eta|) .
\end{aligned}
$$

We will estimate the term with $\beta=0$ :

$$
I=(1+|\xi|+|\eta|)^{(m(\sigma-1)) / \sigma-j / \sigma} \exp \left[-\delta_{1} A \xi^{2}+\delta_{2} A \eta^{2}\right] \cdot \int_{t^{\prime}}^{t}|b(x, s)| d s(|\xi|+|\eta|)
$$

By the assumption (2.5) we have

$$
\begin{aligned}
\int_{t^{\prime}}^{t}|b(x, s)| d s(|\xi|+|\eta|) & \leqq C\left(t-t^{\prime}\right)^{1 / 2} A^{1 / 2}(|\xi|+|\eta|) \\
& \leqq C\left(|\xi|^{-1 / \sigma}+|\eta|^{-1 / \sigma}\right)\left(\left(t-t^{\prime}\right)^{\sigma}\left(\xi^{2}+\eta^{2}\right)^{1 / 2 a}\left(A\left(\xi^{2}+\eta^{2}\right)\right)^{1 / 2}\right)
\end{aligned}
$$

Then we have

$$
\begin{aligned}
& I \leqq C_{1}(1+|\xi|+|\eta|)^{(m(\sigma-1))-(j+1) / \sigma} \exp \left[-\left(\delta_{1}-\varepsilon\right) A \xi^{2}+\left(\delta_{2}+C_{2}\right) A \eta^{2}\right] \\
&\left(0<\varepsilon<\delta_{1}\right) .
\end{aligned}
$$


To estimate the terms with $\beta \geqq 1$ we use the assumption (2.1).

$$
\begin{aligned}
I I & \equiv \sum_{\beta=1}^{\alpha}(1=|\xi|+|\eta|)^{(m-\beta)(\sigma-1) / \sigma j / \sigma} \exp \left[-\delta_{1} A \xi^{2}+\delta_{2} A \eta^{2}\right] \\
& \leqq \\
\leqq & \int_{t^{\prime}}^{t}\left|D_{x}^{\beta} b(x, s)\right| d s(|\xi|+|\eta|) \\
& (t-|\xi|+|\eta|)^{(m-1)(\sigma-1) / \sigma-j / \sigma} \exp \left[-\delta_{1} A \xi^{2}+\delta_{2} A \eta^{2}\right] \cdot\left(t-t^{\prime}\right)(|\xi|+|\eta|), \\
& (\eta \eta \mid) \leqq C(|\xi|+|\eta|)^{1-(2 / \sigma}\left(\left(t-t^{\prime}\right)^{\sigma}\left(\xi^{2}+\eta^{2}\right)\right)^{1 / \sigma} .
\end{aligned}
$$

Thus we have

$$
I I \leqq C_{1}(1+|\xi|+|\eta|)^{(m(\sigma-1)) / \sigma-(j+1) / \sigma} \exp \left[-\left(\delta_{1}-\varepsilon\right) A \xi^{2}+\left(\delta_{2}+C_{2}\right) A \eta^{2}\right] .
$$

Combining the above two estimates we have

$$
I_{m, \alpha}^{(2)} \leqq C(1+|\xi|+|\eta|)^{(m(\sigma-1)) / \sigma-(j+) / \sigma} \exp \left[-\delta_{1} A \xi^{2}+\delta_{2} A \eta^{2}\right],
$$

where $C, \delta_{1}$ and $\delta_{2}$ depend only on $m$ and $j$.

To complete the proof of Lemma,4.2, it remains to estimate the terms :

$$
\begin{aligned}
& D_{x}^{m} \int_{t^{\prime}}^{t} K_{0}(x, \zeta ; t, s) a(x, s) D_{x}^{2} K_{j}\left(x, \zeta ; s, t^{\prime}\right) d s, \\
& D_{x}^{m} \int_{t^{\prime}}^{t} K_{0}(x, \zeta ; t, s) b(x, s) D_{x} K_{j}\left(x, \zeta ; s, t^{\prime}\right) d s, \\
& D_{x}^{m} \int_{t^{\prime}}^{t} K_{0}(x, \zeta ; t, s) c(x, s) K_{j}\left(x, \zeta ; s, t^{\prime}\right) d s
\end{aligned}
$$

which are treated in the same manner as above and we get Lemma 4.2.

Using Lemma 4.2 we shall obtain the precise estimate of $\mathscr{K}_{j}$ :

$$
\mathscr{K}_{j}\left(x, y, t, t^{\prime}\right)=(2 \pi)^{-1 / 2} \int_{-\infty}^{\infty} e^{i(x-y) \xi} K_{j}\left(x, \xi ; t, t^{\prime}\right) d \xi \quad(3.12) .
$$

THEOREM 4.3. For every integers $m, j \geqq 0$ the following inequality holds :

$$
\begin{aligned}
&\left|D_{x}^{m} \mathscr{K}_{j}\left(x, y, t, t^{\prime}\right)\right| \leqq C A^{j / 2 \sigma-(1+m) / 2} \exp \left[\frac{-c(x-y)^{2}}{A}\right], \\
&\left(x, y, t, t^{\prime}\right) \in R \times R \times \Delta
\end{aligned}
$$

where $C$ and $c$ are positive constants depending only on $m$ and $j$.

Proof. We can easily see that $K_{j}\left(x, \zeta ; t, t^{\prime}\right), m, j=0,1, \cdots$, are entire functions in $\zeta, \zeta \in \boldsymbol{C}$. Using Cauchy's theorem and the inequality (4.9), we find that 


$$
\mathscr{K}_{j}\left(x, y, t, t^{\prime}\right)=(2 \pi)^{-1 / 2} \int_{R_{\xi}} e^{i(x-y)(\xi+i \eta)} K_{j}\left(x, \xi+i \eta ; t, t^{\prime}\right) d \xi
$$

is independent of $\eta$, and hence, it coincides with the functions $\mathscr{K}_{j}$ defined in (3.12) for $0 \leqq t^{\prime}<t \leqq T$. We have

$$
\begin{aligned}
& D_{x}^{m} \mathscr{K}_{j}\left(x, y, t, t^{\prime}\right)=(2 \pi)^{-1 / 2} \sum_{\alpha=0}^{m}\left(\begin{array}{c}
m \\
\alpha
\end{array}\right) \\
& \quad \cdot \int_{R_{\xi}} e^{i(x-y)(\xi++\eta)}(i \xi-\eta)^{m-\alpha} D_{x}^{\alpha} K_{j}\left(x, \xi+i \eta ; t, t^{\prime}\right) d \xi, \quad 0 \leqq t^{\prime}<t \leqq T .
\end{aligned}
$$

We shall analyse each term of the right side. Using (4.9) again we set

$$
\begin{aligned}
&\left|\int_{R_{\xi}} e^{i(x-y)(\xi+i \eta)}(i \xi-\eta)^{m-\alpha} D_{x}^{\alpha} K_{j}\left(x, \xi+i \eta ; t, t^{\prime}\right) d \xi\right| \\
& \leqq C e^{-(x-y) \eta} \int_{R_{\xi}}(1+|\xi|+|\eta|)^{m-\alpha+(\alpha(\sigma-1)) / \sigma-j / \sigma} \exp \left[-\delta_{1} A \xi^{2}+\delta_{2} A \eta^{2}\right] d \xi \\
& \leqq C_{1} e^{-(x-y) \eta} \exp \left[\delta_{2} A \eta^{2}\right] \int_{R_{\xi}}(1+|\xi|)^{m-j / \sigma} \exp \left[-\delta_{1} A \xi^{2}\right] d \xi \\
&+C_{2} e^{-(x-y) \eta}|\eta|^{m-j / \sigma} \exp \left[\delta_{2} A \eta^{2}\right] \int_{R_{\xi}} \exp \left[-\delta_{1} A \xi^{2}\right] d \xi \\
& \equiv I+I I .
\end{aligned}
$$

Take

$$
\eta=\frac{x-y}{2 \delta_{2} A\left(x, t, t^{\prime}\right)}, \quad\left(x, y, t, t^{\prime}\right) \in R \times R \times \Delta .
$$

Then we obtain

$$
I \leqq C_{1}^{\prime} A^{j / 2 \sigma-1 / 2(1+m)} \exp \left[\frac{-(x-y)^{2}}{4 \delta_{2} A}\right] .
$$

As for $I I$ we have

$$
\begin{aligned}
I I & \leqq C^{\prime} A^{-1 / 2} \exp \left[-\frac{(x-y)^{2}}{4 \delta_{2} A}\right]\left(\frac{x-y}{2 \delta_{2} A}\right)^{m-j / \sigma} \\
& =C^{\prime} A^{-1 / 2} \exp \left[\frac{(x-y)^{2}}{4 \delta_{2} A}\right]\left(\frac{x-y}{2 \delta_{2} A^{1 / 2}}\right)^{m-j / \sigma} A^{-1 / 2(m-j / \sigma)} \\
& \leqq C^{\prime \prime} A^{j / 2 \sigma-1 / 2(1+m)} \exp \left[-\frac{(x-y)^{2}}{5 \delta_{2} A}\right]
\end{aligned}
$$

\section{\&5. Construction of the fundamental solution.}

As in the book [2], we give the following definition. 
DeFinition 5.1. A fundamental solution of $P u=0$ in $R_{x} \times I$ is a function $\Gamma\left(x, y, t, t^{\prime}\right)$ defined in $R_{x} \times R_{y} \times \Delta$ which satisfies the following conditions :

(i) for fixed $\left(y, t^{\prime}\right), \Gamma$ is, as a function of $(x, t)\left(x \in R, 0 \leqq t^{\prime}<t \leqq T\right)$, a solution of the equation $P u=0$;

(ii) it holds that

$$
\lim _{t \downarrow t^{\prime}} \int_{R_{y}} \Gamma\left(x, y, t, t^{\prime}\right) \varphi(y) d y=\varphi(x), \quad x \in R_{x},
$$

for every continuous function $\varphi(x)$ such that

$$
|\varphi(x)| \leqq \text { const. } \exp \left[h x^{2}\right], \quad h>0 .
$$

LEMMA 5.1. Let $\varphi(x)$ be a continuous function satisfying (5.2) with some positive constant $h$. Then we have

$$
\begin{gathered}
\lim _{t \downarrow t^{\prime}} \int_{R_{y}} \mathscr{K}_{0}\left(x, y, t, t^{\prime}\right) \varphi(y) d y=\varphi(x), \quad x \in R_{x}, \\
\lim _{t \downarrow t^{\prime}} \int_{R_{y}} \mathscr{K}_{j}\left(x, y, t, t^{\prime}\right) \varphi(y) d y=0, x \in R_{x}, \quad j=1,2, \cdots .
\end{gathered}
$$

Proof. We can easily see that

$$
\mathscr{K}_{0}\left(x, y, t, t^{\prime}\right)=\frac{1}{2 \sqrt{\pi A}} \exp \left[-\frac{(x-y)^{2}}{4 A}\right] .
$$

Then it follows that

$$
\int_{-\infty}^{\infty} \mathscr{K}_{0}\left(x, y, t, t^{\prime}\right) d y=1
$$

Thus we have

$$
\begin{aligned}
\int_{-\infty}^{\infty} \mathscr{K}_{0}\left(x, y, t, t^{\prime}\right) \varphi(y) d y & =\varphi(x)+\int_{-\infty}^{\infty} \mathscr{K}_{0}\left(x, y, t, t^{\prime}\right)(\varphi(y)-\varphi(x)) d y \\
& \equiv \varphi(x)+I .
\end{aligned}
$$

The integral $I$ is divided into two parts: $I_{1}$ with $|y-x|<\delta$ and $I_{2}$ with $|y-x| \geqq \delta$, and take $\delta$ such that $|f(y)-f(x)|<\varepsilon$ if $|y-x|<\delta$. Here $\varepsilon$ is any fixed positive number, and $\delta$ is a fixed positive number depending on $\varepsilon$. If we use the estimate (4.13) we obtain

$$
\int_{-\infty}^{\infty}\left|\mathscr{K}_{0}\left(x, y, t, t^{\prime}\right)\right| d y \leqq C \quad 0 \leqq t^{\prime}<t \leqq T,
$$


where $C$ is independent of $t, t^{\prime}$. Similarly we see that

$$
\int_{|y-x| \geq \delta}\left|\mathscr{K}_{0}\left(x, y, t, t^{\prime}\right)\right| \exp \left[2 h(x-y)^{2}\right] d y \rightarrow 0 \quad t \downarrow t^{\prime} .
$$

Using (5.4) it follows that

$$
\left|I_{1}\right| \leqq \varepsilon \int_{|y-x|<\delta}\left|\mathscr{K}_{0}\left(x, y, t, t^{\prime}\right)\right| d \xi \leqq C \cdot \varepsilon .
$$

Using (5.6) and the inequality

$$
|\varphi(y)| \leqq \text { const. } \exp \left[2 h(x-y)^{2}\right],
$$

where the constant depends on $x$, it is proved that $I_{2} \rightarrow 0$ as $t \downarrow t^{\prime}$ (for each fixed $x$ ). Hence $\left|I_{2}\right|<\varepsilon$ if $t-t^{\prime}$ is sufficiently small. Combining this with (5.7) we get $|I| \leqq(C+1) \varepsilon$ if $t-t^{\prime}$ is sufficiently small. Since $\varepsilon$ is arbitrary, (5.3) follows.

To prove (5.4), using the inequalities (4.13) and (5.8) we have, for any fixed $x \in R$,

$$
\begin{aligned}
\left|\int\right| \mathscr{K}_{j}\left(x, y, t, t^{\prime}\right) \varphi(y) d y \mid & \leqq C_{x} A^{j / 2 \sigma} \int A^{-1 / 2} \exp \left[\frac{c_{x}(x-y)^{2}}{A}\right] d y \\
& \leqq C^{\prime} A\left(x, t, t^{\prime}\right)^{j / 2 \sigma} \rightarrow 0 \text { as } t \downarrow t^{\prime}, \quad j=1,2, \cdots,
\end{aligned}
$$

Now we set

$$
E_{\mu}=\sum_{j=0}^{\mu} \mathscr{K}_{j}\left(x, y, t, t^{\prime}\right), \quad \mu=0,1,2, \cdots
$$

Then we have

$$
\begin{aligned}
P_{x, t} E_{\mu} & =\delta\left(x-y, t-t^{\prime}\right)+(2 \pi)^{-1 / 2} \int_{-\infty}^{\infty} e^{i(x-y) \xi} L_{2} K_{\mu}\left(x, \xi ; t, t^{\prime}\right) d \xi \\
& \equiv \delta\left(x-y, t-t^{\prime}\right)+F_{\mu}\left(x, y, t, t^{\prime}\right), \quad(\S 3, \text { (i)) } .
\end{aligned}
$$

LEMMA 5.2. We have

$$
\begin{array}{r}
\left|F_{\mu}\left(x, y, t, t^{\prime}\right)\right| \leqq C A^{-3 / 2+\mu / 2 \sigma} \exp \left[-\frac{c(x-y)^{2}}{A}\right], \quad \mu=0,1,2, \cdots, \\
\left(x, y, t, s^{\prime}\right) \in R \times R \times \Delta,
\end{array}
$$

where the constants $C$ and $c$ depend only on $\mu$.

Proof. If we substitute $\xi$ by $\zeta=\xi+i \eta \in C$, then $L_{2} K_{\mu}\left(x, \zeta ; t, t^{\prime}\right)$ is an entire function of $\zeta$. By Lemma 4.2 we have 


$$
\left|L_{2} K_{\mu}\left(x, \zeta ; t, t^{\prime}\right)\right|=C(1+|\xi|+|\eta|)^{2-\mu / \sigma} \exp \left[-\delta_{1} A \xi^{2}+\delta_{2} A \eta^{2}\right],
$$

where the constants $C, \delta_{1}$ and $\delta_{2}$ depend only on $\mu$. Using Cauchy's theorem and setting $\eta=(x-y) / 2 \delta_{2} A\left(x, t, t^{\prime}\right)$ as in the proof of Theorem 4.3, we have the inequality (5.9).

We shall need the following lemmas.

Lemma 5.3. (cf. [2], Ch. 1. Lemma 1.) Let $f(x, y)$ be a continuous function of $(x, y)$ when $x, y$ bury in a compact domain $S$ of $R_{x}^{m} \times R_{y}^{m}$ and $x \neq y$, and let

$$
\int_{S(x, \boldsymbol{s})}|f(x, y)| d y \rightarrow 0 \quad \text { as } \varepsilon \rightarrow 0
$$

uniformly with respect to $x$ in $S$, where $S(x, \varepsilon)$ is the intersection of $S$ with the ball with center $x$ and radius $\varepsilon$. Then, for any bounded measurable function $g(y)$ in $S$, the (improper) integral

$$
h(x)=\int_{S} f(x, y) g(y) d y
$$

is a continuous function in $S$.

LEMMA 5.4. Let $f(x, t)$ be a measurable function in $R \times I_{0}, I_{0}=$ $\left[T_{0}, T_{1}\right], 0 \leqq T_{0}<T_{1} \leqq T$, satisfying

$$
|f(x, t)| \leqq \text { const. } \exp \left[\lambda x^{2}\right] \text { a.e. in } R \times I_{0} \quad \text { for } \lambda \leqq c\left(T_{1}-T_{0}\right)^{-\sigma}
$$

where $c$ is chosen depending only on $\mu$.

Then

$$
\begin{gathered}
\Phi(x, t)=\int_{T_{0}}^{t} \int_{R_{y}} E_{\mu}\left(x, y, t, t^{\prime}\right) f\left(y, t^{\prime}\right) d y d t^{\prime}, \quad \mu=0,1,2, \cdots, \\
\Psi(x, t)=\int_{T_{0}}^{t} \int_{R_{y}} F_{\mu}\left(x, y, t, t^{\prime}\right) f\left(y, t^{\prime}\right) d y d t^{\prime}, \quad \mu \geqq 2 \sigma
\end{gathered}
$$

are continuous functions in $R \times I_{0}$ and

$$
\lim _{t \downarrow T_{0}} \Phi(x, t)=0, \quad x \in R_{x} .
$$

Furthermore, for $\mu \geqq \sigma / 2$ we have the following equality in the distribution sense:

$$
P \Phi(x, t)=f(x, t)+\Psi(x, t) \quad \text { in } R \times\left(T_{0}, T_{1}\right) .
$$


Proof. We note that $\Phi(x, t)$ is called the volume potential of $f$ with respect to the parametrix $E_{\mu}$. This is an improper integral, the integrand having a singularity at $y=x, t^{\prime}=t$. However the singularity is integrable. Indeed, by Theorem 4.3 , we have

$$
\left|E_{\mu}\left(x, y, t, t^{\prime}\right)\right| \leqq \text { const. } A^{-1 / 2} \exp \left[-\frac{c_{\mu}(x-y)^{2}}{A}\right] .
$$

Then we have

$$
\int_{-\infty}^{\infty}\left|E_{\mu}\left(x, y, t, t^{\prime}\right)\right| d y \leqq \text { const. } c_{\mu}{ }^{1 / 2} .
$$

Hence the singularity is integrable.

If

$$
\lambda<\frac{c_{\mu}}{c\left(T_{1}-T_{0}\right)}
$$

then $\lambda<\left(c_{u}-\varepsilon\right) / A$ by the assumption (2.3), and the integral in (5.11) exists.

The continuity of $\Phi(x, t)$ follows by breaking the $y$-integral into two parts and treating each part separately. The continuity of the integral corresponding to the unbounded part $D_{1}$ of $R_{y}$ ( $x$ is bounded away from $D_{1}$ ) follows by a standard theorem of calculus, whereas the continuity of the integral corresponding to the bounded part $D_{0}$ of $R_{y}$ follows by employing Lemma 5.3.

The continuity of $\psi(x, t)$ is obtained similarly as above taking the bound of $\lambda$ smaller than (5.16) if necessary. Indeed, we have by (5.9)

$$
\left|F_{\mu}\left(x, y t, t^{\prime}\right)\right| \leqq C_{\mu}^{\prime} A^{-1 / 2} \exp \left[-\frac{c_{\mu}(x-y)^{2}}{A}\right]
$$

if $\mu \geqq 2 \sigma$ and the singularity is integrable as above.

Remembering that

$$
E_{\mu}\left(x, y, t, t^{\prime}\right)=0 \quad t<t^{\prime},
$$

we have (5.13) by the same consideration as above.

It remains to prove the equality (5.14). By the property (i) of $\S 3$ it follows that if $\mu \geqq 2 \sigma$ 


$$
\begin{aligned}
& P_{x, t} \int_{T_{0}}^{t} \int_{R_{y}} E_{\mu}\left(x, y, t, t^{\prime}\right) \varphi\left(y, t^{\prime}\right) d y d t^{\prime} \\
& \quad=\varphi(x, t)+\int_{T_{0}}^{t} \int_{R_{y}} F_{\mu}\left(x, y, t, t^{\prime}\right) \varphi\left(y, t^{\prime}\right) d y d t^{\prime}
\end{aligned}
$$

for any $\varphi(x, t) \in C_{0}^{\infty}\left(R \times\left[T_{0}, T_{1}\right]\right)$. By continuity this equality holds in the distribution sense for any bounded measurable function $\varphi(x, t)$ with compact support in $R \times I_{0}$. For $f(x, t)$ as given in Lemma 5.4, we set

$$
f_{n}(x, t)=\left\{\begin{array}{cl}
f(x, t) & |x| \leqq n, \\
0 & |x|>n, \quad n=1,2, \cdots .
\end{array}\right.
$$

Then we find that

$$
\begin{aligned}
f_{n}(x, t) \rightarrow f(x, t) & \\
\Phi_{n}(x, t) & =\int_{T_{0}}^{t} \int_{R_{y}} E_{\mu}\left(x, y, t, t^{\prime}\right) f_{n}\left(y, t^{\prime}\right) d y d t^{\prime} \rightarrow \Phi(x, t), \\
\Psi_{n}(x, t) & =\int_{T_{0}}^{t} \int_{R_{y}} F_{\mu}\left(x, y, t, t^{\prime}\right) f_{n}\left(y, t^{\prime}\right) d y d t^{\prime} \rightarrow \Psi(x, t)
\end{aligned}
$$

in the distribution sense as $n \rightarrow \infty$. Thus we obtain the equality (5.14) and this completes the proof of Lemma 5.4.

Now applying the parametrix method (cf. [2], Ch. 9), we shall construct the fundamental solution $\Gamma$ in the form

$$
\Gamma\left(x, y, t, t^{\prime}\right)=E_{\mu}\left(x, y, t, t^{\prime}\right)+\int_{t^{\prime}}^{t} d s \int_{R_{z}} E_{u}(x, z, t, s) \Phi\left(z, y, s, t^{\prime}\right) d z
$$

when $\mu \geqq 3 \sigma$.

If $\Phi$ is a function such that Lemma 5.4 can be applied to the integral on the right hand side of (5.18), then $\Gamma$ satisfies the equation $P \Gamma=0$ as a function of $(x, t)$ (see (i) of Def. 5.1) if and only if

$$
\begin{aligned}
& \Phi\left(x, y, t, t^{\prime}\right) \\
& \quad=-F_{\mu}\left(x, y, t, t^{\prime}\right)-\int_{t^{\prime}}^{t} d s \int_{R_{z}} F_{\mu}(x, z, t, s) \Phi\left(z, y, s, t^{\prime}\right) d z\left(t>t^{\prime}\right) .
\end{aligned}
$$

The following series is a formal solution of (5.19):

$$
\Phi\left(x, y, t, t^{\prime}\right)=\sum_{m=1}^{\infty} \Phi_{m}\left(x, y, t, t^{\prime}\right),
$$

where $\Phi_{1}=F_{\mu}$ and

$$
\Phi_{m}\left(x, y, t, t^{\prime}\right)=\int_{t^{\prime}}^{t} d s \int_{R_{z}} \Phi_{1}(x, z, t, s) \Phi_{m-1}\left(z, y, s, t^{\prime}\right) d z, \quad m \geqq 2 .
$$


Setting $a_{\mu}=\frac{1}{2}\left(\frac{\mu}{\sigma}-3\right), \mu \geqq 3 \sigma$, we have by (5.9)

$$
\left|F_{\mu}\right| \leqq c_{1}\left(t-t^{\prime}\right)^{a_{\mu}} \exp \left[\frac{-c_{2}(x-y)^{2}}{\left(t-t^{\prime}\right)}\right]
$$

where the constants $c_{1}$ and $c_{2}$ depend only on $\mu$. Thus $F_{\mu}=\Phi_{1}$ is a bounded function if $\mu \geqq 3 \sigma$.

We shall prove by induction in $m$ that

$$
\begin{aligned}
\left|\Phi_{m+1}\left(x, y, t, t^{\prime}\right)\right| \leqq C_{1} A_{1}^{m}\left(t-t^{\prime}\right)^{m\left(a_{\mu}+1\right)} \frac{1}{m !} \exp \left[\frac{A_{2}(x-y)^{2}}{\left(t-t^{\prime}\right)}\right] & \\
0 & <A_{2}<c_{2} .
\end{aligned}
$$

Indeed, (5.22) implies (5.23) the case $m=0$. Assume (5.23) for $m \geqq 0$, then we have

$$
\begin{array}{r}
\left|\Phi_{m+2}\right|=\frac{C_{1}^{2} A_{1}^{m}}{m !} \int_{t^{\prime}}^{t} \mathrm{ds} \int_{R_{z}}(t-s)^{a_{\mu}} \exp \left[\frac{-c_{2}(x-z)^{2}}{t-s}\right]\left(s-t^{\prime}\right)^{m\left(a_{\mu}+1\right)} \\
\cdot \exp \left[\frac{A_{2}(z-y)^{2}}{s-t^{\prime}}\right] d z \\
\leqq \frac{C_{1}^{2} A_{1}^{m}}{m !}\left(t-t^{\prime}\right)^{a_{\mu}} \int_{t^{\prime}}^{t}\left(s-t^{\prime}\right)^{m\left(a_{\mu}+1\right)} d s \int_{R_{z}} \exp \left[-\frac{\left(c_{2}-A_{2}\right)(x-z)^{2}}{t-s}\right] \\
\cdot \exp \left[-A_{2}\left(\frac{(x-z)^{2}}{t-s}+\frac{(z-y)^{2}}{s-t^{\prime}}\right)\right] d z
\end{array}
$$

We can easily see

$$
\min _{s, t}\left[\frac{(x-z)^{2}}{t-s}+\frac{(z-y)^{2}}{s-t^{\prime}}\right]=\frac{(x-y)^{2}}{t-t^{\prime}} .
$$

Thus we have

$$
\begin{aligned}
&\left|\Phi_{m+2}\right| \leqq \frac{C_{1}^{2} A_{1}^{m}}{m !}\left(t-t^{\prime}\right)^{a_{\mu}} \cdot \exp \left[-\frac{A_{2}(x-y)^{2}}{t-t^{\prime}}\right] \cdot \int_{t^{\prime}}^{t}\left(s-t^{\prime}\right)^{m\left(a_{\mu}+1\right)} d s \int_{R_{z}} \\
& \cdot \exp \left[-\frac{\left(c_{2}-A_{2}\right)(x-z)^{2}}{t-s}\right] d z \\
& \leqq \frac{C_{1} A_{1}^{m} c_{1} C T^{1 / 2}}{\left(m !\left(m\left(a_{\mu}+1\right)+1\right)\right.}\left(t-t^{\prime}\right)^{(m+1)\left(a_{\mu}+1\right)} \exp \left[-\frac{A_{2}(x-y)^{2}}{t-t^{\prime}}\right] .
\end{aligned}
$$

If we take $A_{1}=\max \left(c_{1}, c_{2} C T^{1 / 2}\right)$, the proof of (5.23) is completed.

From (5.23) it follows that the series in (5.20) is convergent and 
that $\Phi$ satisfies (5.19). Furthermore, from the estimates of $\Phi_{m}(m \geqq 1)$ we see that

$$
\left|\Phi\left(x, y, t, t^{\prime}\right)\right| \leqq A_{1}^{\prime}\left(t-t^{\prime}\right)^{a}{ }_{\mu} \exp \left[-\frac{A_{2}(x-y)^{2}}{t-t^{\prime}}\right],
$$

where the constants $A_{1}^{\prime}$ and $A_{2}$ depend only on $\mu$. It is then clear that Lemma 5.4 is applied to the integral on the right hand side of (5.18) and thus $\Gamma$ satisfies the equation $P_{x, t} \Gamma\left(x, y, t, t^{\prime}\right)=0\left(0 \leqq t^{\prime}<t \leqq T\right)$.

The second property of fundamental solution, namely (5.1), follows from Lemma 5.1 and the following estimate:

$$
\left|\int_{t^{\prime}}^{t} d s \int_{R_{z}} E_{\mu}(x, y, t, s) \Phi\left(z, y, s, t^{\prime}\right) d z\right| \leqq C_{1}\left(t-t^{\prime}\right)^{a_{\mu}} \exp \left[-\frac{C_{2}(x-y)^{2}}{t-t^{\prime}}\right]
$$

where the constants $C_{1}$ and $C_{2}$ depend only on $\mu$. We have (5.25) as follows :

$$
\begin{gathered}
\left|\int_{t^{\prime}}^{t} d s \int_{R_{z}} E_{\mu} \Phi d z\right| \leqq \text { const. }\left(t-t^{\prime}\right)^{a_{\mu}} \cdot \int_{t^{\prime}}^{t} d s \int_{R_{z}}(t-s)^{-\sigma / 2} \\
\quad \cdot \exp \left[-\frac{C^{\prime}(x-y)^{2}}{t-s}-\frac{C^{\prime \prime}(z-y)^{2}}{s-t^{\prime}}\right] \quad \text { (Th. 4.3) } \\
\leqq \text { const. }\left(t-t^{\prime}\right)^{a_{\mu}} \cdot \exp \left[-\frac{\text { const. }(x-y)^{2}}{t-t^{\prime}}\right] \\
\quad \cdot \int_{t^{\prime}}^{t}(t-s)^{\sigma^{\prime 2(\gamma-1)}} d s \int_{R_{z}}(x-z)^{-\gamma} \exp \left[\frac{\text { const. }(x-z)^{2}}{t-s}\right] d z \\
\leqq \\
\quad C_{1}\left(t-t^{\prime}\right)^{a_{\mu}} \exp \left[\frac{C_{2}(x-y)^{2}}{t-t^{\prime}}\right] .
\end{gathered}
$$

Thus we have obtained the following theorem.

THEOREM 5.5. There exists a fundamental solution $\Gamma\left(x, y, t, t^{\prime}\right)$ (given in (5.18)) of $P u=0$ in $R_{x} \times I$ satisfying the inequality:

$$
\begin{aligned}
&\left|\Gamma\left(x, y, t, t^{\prime}\right)\right| \leqq C A^{-1 / 2} \exp \left[-\frac{c(x-y)^{2}}{A}\right]+ C^{\prime}\left(t-t^{\prime}\right)^{a_{\mu}} \\
& \cdot \exp \left[-\frac{c^{\prime}(x-y)^{2}}{t-t^{\prime}}\right],
\end{aligned}
$$

where 


$$
A=\int_{t^{\prime}}^{t} a(x, \tau) d \tau, \quad a_{\mu}=\frac{1}{2}\left(\frac{\mu}{\sigma}-3\right) ; \mu \geqq 3 \sigma,
$$

and the constants $C, c, C^{\prime}$ and $c^{\prime}$ depend only on $\mu$.

\section{§ 6. Existence of solution.}

THEOREM 6.1. (Existence) Let $f(x, t)$ be a measurable function in $R \times I$ and $\varphi(x)$ be a continuous function in $R$ and assume that

$$
\begin{gathered}
|f(x, t)| \leqq M \exp \left[\mathrm{k} x^{2}\right] \quad \text { a.e. } \quad \text { in } R \times I, \\
|\varphi(x)| \leqq M \exp \left[k x^{2}\right] \quad \text { in } R .
\end{gathered}
$$

Then the function

$$
u(x, t)=\int_{0}^{t} \int_{R_{y}} \Gamma\left(x, y, t, t^{\prime}\right) f\left(y, t^{\prime}\right) d y d t^{\prime}+\int_{R_{y}} \Gamma(x, y, t, 0) \varphi(y) d y
$$

is a solution of the Cauchy problem (2.6), (2.7) in the strip $0<t<t_{0}$ where $t_{0}=\min \left(T, \frac{\bar{c}}{k}\right)$ and where $\bar{c}$ is a constant depending on $P$, and

$$
|u(x, t)| \leqq \text { const. } \exp \left[k^{\prime} x^{2}\right] \quad x \in R, \quad 0 \leqq t \leqq t_{0},
$$

for some constant $k^{\prime}$. The solution $u(x, t)$ is in $C^{\infty}\left(R \times\left(0, t_{0}\right]\right)$ if $f(x, t)$ is in $C^{\infty}(R \times I)$.

We prepare the following simple lemma whose proof is omitted.

LEMMA 6.2. For any positive numbers $A$ and $B$ with $B<\frac{1}{3} A$, there exists a positive constant $C$ such that, for all $x \in R$,

$$
\int_{R_{y}} \exp \left[-A(x-y)^{2}\right] \exp \left[B y^{2}\right] d y \leqq C A^{-1 / 2} \exp \left[\frac{2}{3} A x^{2}\right] .
$$

Proof of Theorem 6.1. First we consider the function

$$
u_{1}(x, t)=\int_{0}^{t} \int_{R_{y}} \Gamma\left(x, y, t, t^{\prime}\right) f\left(y, t^{\prime}\right) d y d t^{\prime}
$$

By (5.18), decompose $u_{1}(x, t)$ into two parts;

$$
\begin{aligned}
u_{1}(x, t)= & \int_{0}^{t} \int_{R_{y}} E_{\mu}\left(x, y, t, t^{\prime}\right) f\left(y, t^{\prime}\right) d y d t^{\prime} \\
& +\int_{0}^{t} \int_{R_{y}}\left[\int_{t^{\prime}}^{t} \int_{R_{z}} E_{\mu}(x, z, t, s) \Phi\left(z, y, s, t^{\prime}\right) d z d s\right] f\left(y, t^{\prime}\right) d y d t^{\prime} \\
\equiv & I+I I .
\end{aligned}
$$


By Lemma 5.4 we are given

$$
P_{x, t} I=f(x, t)+\int_{0}^{t} \int_{R_{y}} F_{\mu}\left(x, y, t, t^{\prime}\right) f\left(y, t^{\prime}\right) d y d t^{\prime}
$$

in a strip $0<t<t_{1}$. And by using the estimates (5.10), (5.15) and Lemma 6.2 we have

$$
|I| \leqq \text { const. } \exp \left[\text { const. } x^{2}\right], \quad 0 \leqq t \leqq t_{1} .
$$

Next we analyse the integral $I I$. Define $\Phi\left(x, y, t, t^{\prime}\right)=0$ for $t<t^{\prime}$. Then after changing the order of integration the integral $I I$ can be written in the form:

$$
I I=\int_{0}^{t} \int_{R_{z}} E_{\mu}(x, z, t, s)\left[\int_{0}^{t} \int_{R_{y}} \Phi\left(z, y, s, t^{\prime}\right) f\left(y, t^{\prime}\right) d y d t^{\prime}\right] d z d s .
$$

By Lemma 6.2 and by the estimate (5.23) we have

$$
\left|\int_{0}^{t} \int_{R_{y}} \Phi\left(z, y, s, t^{\prime}\right) f\left(y, t^{\prime}\right) d y d t^{\prime}\right| \leqq C_{1} \exp \left[C_{2} z^{2}\right]
$$

in a strip $0<s<t_{2}$.

Thus by Lemma 5.4, the integral $I I$ has a meaning in some strip $0 \leqq$ $t \leqq t_{3}$; in fact this is a continuous function in the strip $0 \leqq t \leqq t_{3}$ by the same reasoning as in the proof of Lemma 5.4, and we have the following equality in the distribution sense:

$$
\begin{aligned}
P_{x, t} I I= & \int_{0}^{t} \int_{R_{y}} \Phi\left(x, y, t, t^{\prime}\right) f\left(y, t^{\prime}\right) d y d t^{\prime} \\
& +\int_{0}^{t} \int_{R_{y}} F_{\mu}(x, z, t, s)\left[\int_{0}^{t} \int_{R_{y}} \Phi\left(z, y, s, t^{\prime}\right) f\left(y, t^{\prime}\right) d y d t\right] d z d s, \\
& 0<t<t_{4}, \quad t_{4}=\min \left(t_{1}, t_{2}, t_{3}\right) .
\end{aligned}
$$

Furthermore, by (5.22) and by using Lemma 6.2 again, we have

$$
\left.|I I| \leqq \text { const. } \exp \text { [const. } x^{2}\right] \quad 0 \leqq t \leqq t_{4} .
$$

Summing up the above considerations we have

$$
\begin{gathered}
P_{x, t} u_{1}(x, t)=f(x, t) \quad \text { in } R \times\left(0, t_{4}\right), \\
\left|u_{1}(x, t)\right| \leqq \text { const. } \exp \left[\text { const. } x^{2}\right] \quad \text { in } R \times\left[0, t_{4}\right] .
\end{gathered}
$$

By (5.26) and by the similar way to the proof of Lemma 5.4 we have

$$
\lim _{t \downarrow 0} u_{1}(x, t)=0 \text {. }
$$


Now we consider the function

$$
u_{2}(x, t)=\int_{R_{y}} \Gamma(x, y, t, 0) \varphi(y) d y .
$$

By using Lemma 6.2, we have

$$
\left.\left|u_{2}(x, t)\right| \leqq \text { const. } \exp \text { [const. } x^{2}\right]
$$

in some strip $0 \leqq t \leqq t_{5}$. By the properties of fundamental solution derived in $\S 5$, we can easily see that $u_{2}(x, t)$ is a continuous function in the strip $0 \leqq t \leqq t_{3}$ and

$$
\lim _{t \downarrow 0} u_{2}(x, t)=\varphi(x) \quad x \in R .
$$

Furthermore, we can easily see that $u_{2}(x, t)$ satisfies the equation

$$
P u_{2}(x, t)=0 \quad \text { in } R \times\left(0, t_{5}\right)
$$

in the distribution sense, hence in the usual sense by virtue of hypoellipticity of $P$. Combining (6.10), (6.12), (6.16) and (6.15) it follows that $u(x, t)$ defined in (6.1) is a solution of the Cauchy problem (2.6), (2.7) in a strip $0 \leqq t \leqq t_{0}, t_{0}=\min \left(t_{4}, t_{5}\right)$. We note that the constants $t_{j}, 1 \leqq j \leqq 5$, are chosen in the form $\min \left\{T, C_{j}^{*} / k\right\}$ where $C_{j}^{*}$ is a constant depending on $P$ (and $\mu$ ) so the same is true for $t_{0}$.

Finally, by the hypoellipticity of $P$ we have $u(x, t) \in C^{\infty}\left(R \times\left(0, t_{0}\right]\right)$ if $f(x, t) \in C^{\infty}(R \times I)$. In this case, we have $u(x, t) \in C\left(R \times\left[0, t_{0}\right]\right) \cap$ $C^{\infty}\left(R \times\left(0, t_{0}\right]\right)$ Q.E.D.

§. Uniqueness of solution. As stated in the introduction we shall follow the method of [1]. Let $\Omega$ be an open finite interval in $R_{x}=$ $\{x ;-\infty<x<\infty\}$. We set $Q=\Omega \times(0, T), \quad \partial_{p} Q=\{\partial \Omega \times[0, T]\} \cup$ $\{\Omega \times(t=0)\}$ and $\tilde{Q}=\bar{Q} / \partial_{p} Q$.

THEOREM 7.1. (Maximum principle.) Let $P$ be the parabolic operator given in §2. Let $u(x, t)$ be a real valued function in $C_{x, t}^{2,1}(\tilde{Q}) \cap C(Q)$ satisfying

$$
\begin{aligned}
& P_{u} \leqq 0 \quad \text { in } \tilde{Q}, \\
& u \leqq 0 \text { on } \partial_{p} Q \text {. }
\end{aligned}
$$

Then we have $u(x, t) \leqq 0$ in $\bar{Q}$. 
Proof. (a) The case $c(x, t)>0$ in $\bar{Q}$. Assume that there were a point $\left(x_{0}, t_{0}\right) \in \tilde{Q}$ such that

$$
\max _{\bar{Q}} u=u\left(x_{0}, t_{0}\right)>0 .
$$

Then the following must hold at $\left(x_{0}, t_{0}\right)$ :

$$
\frac{\partial u}{\partial x}=0, \quad \frac{\partial u}{\partial t} \geqq 0, \quad c u>0 \text {. }
$$

And then

$$
\left.P u\right|_{\left(x_{0}, t_{0}\right)}=\frac{\partial u}{\partial t}\left(x_{0}, t_{0}\right)-\alpha\left(x_{0}, t_{0}\right) \frac{\partial^{2} u}{\partial x^{2}}+c\left(x_{0}, t_{0}\right) u\left(x_{0}, t_{0}\right)>0,
$$

which is a contradiction.

(b) If $c(x, t) \geqq-\gamma$ in $\bar{Q}$ for some $\gamma>0$, we can reduce to the case $c(x, t)>0$. Setting $u(x, t)=e^{\gamma^{\prime} t} v$ with any $\gamma^{\prime}$ larger than $\gamma$, we have

$$
\begin{gathered}
P v+\gamma^{\prime} v \geqq 0 \quad \text { in } \tilde{Q}, \\
v \leqq 0 \quad \text { on } \partial_{p} Q, \\
c(x, t)+\gamma^{\prime}>0 \quad \text { in } \bar{Q},
\end{gathered}
$$

from which our assertion follows immediately.

COROLlaRY 7.2. Let $u(x, t)$ be a real valued function in $C_{x, t}^{2,1}(\tilde{Q}) \cap$ $C(Q)$ satisfying

$$
\begin{aligned}
& P u=0 \quad \text { in } \tilde{Q}, \\
& u=0 \quad \text { on } \partial_{p} Q
\end{aligned}
$$

Then $u(x, t)=0$ in $\bar{Q}$.

THEOREM 7.3. (cf. [1], Theorem 1.) Let $u(x, t)$ be a complex valued function in $C_{x, t}^{2,1}(R \times(0, T]) \cap C(R \times[0, T])$ satisfying

$$
\begin{gathered}
P u=0 \quad \text { in } R \times(0, T], \\
u(x, 0)=0 \quad \text { on } R, \\
|u(x, t)| \leqq M \exp \left[k\left(x^{2}+1\right)\right] \quad \text { in } R \times[0, T], M, k>0 .
\end{gathered}
$$

Then we have $u(x, t)=0$ in $R \times[0, T]$.

Proof. We can easily see that it is sufficient to prove the theorem in the case where $u(x, t)$ is a real value function. 
We set $v=\exp \left[2 k e^{\theta t}\left(x^{2}+1\right)\right]$ with $\theta>0$ determined later. Then we have

$$
\begin{aligned}
\frac{P v}{v} & =4 k e^{\theta t} \theta\left(x^{2}+1\right)-16 k^{2} e^{2 \theta t} x^{2} a(x, t)+4 k e^{\theta t}(a(x, t)+x b(x,))+c(x, t) \\
& \geqq 4 k e^{\theta t}\left(x^{2}+1\right)\left(\theta-C_{1} k e^{\theta t}-C_{2}\right) \\
& \left.\geqq 4 k e^{\theta t}\left(x^{2}+1\right) \theta-C_{1} k e-C_{2}\right), \quad 0 \leqq t \leqq \theta^{-1}
\end{aligned}
$$

where the constants $C_{1}$ and $C_{2}$ depend only on $\max _{R \times I}(|a(x, t)|+|b(x, t)|+$ $|c(x, t)|)$. If we put $\theta=2 H, H=C_{1} k e+C_{2}$, then we have

$$
P v>0 \quad \text { in } R \times\left[0, H^{-1}\right] .
$$

Next, for any $\rho>0$, we set

$$
\begin{aligned}
& w=u-M \exp \left[2 k e^{H t}\left(x^{2}+1\right)-k\left(\rho^{2}+1\right)\right] \\
& \text { in }(|x|<\rho) \times\left[0, H^{-1}\right] \equiv Q_{\rho} .
\end{aligned}
$$

Then we have

$$
P w=P u-M \exp \left[-k\left({ }^{2}+1\right) P v<0 \quad \text { in } \tilde{Q}, \quad w(x, t) \leqq 0 \quad \text { on } \partial_{p} Q .\right.
$$

By Theorem 7.1, we have $w(x, t) \leqq 0$ in $\bar{Q}_{\rho}$. For any point $\left(x^{*}, t^{*}\right) \in R$ $\times\left[0, H^{-1}\right]$, we have $\left(x^{*}, t^{*}\right) \in \bar{Q}_{\rho}$ if we take $\rho$ sufficiently large, and hence we have $w\left(x^{*}, t^{*}\right) \leqq 0$. Thus we have

$$
u\left(x^{*}, t^{*}\right) \leqq M \exp \left[-k\left(\rho^{2}+1\right)\right] v\left(x^{*}, t^{*}\right) .
$$

Since $\rho$ is arbitrary and the right hand side tends to zero as $\rho \rightarrow \infty$, we have

$$
u(x, t) \leqq 0 \quad \text { in } R \times\left[0, H^{-1}\right] .
$$

Iterating this procedure finitely many times, we obtain

$$
u(x, t) \leqq 0 \quad \text { in } R \times[0, T) .
$$

Similarly we have

$$
u(x, t) \geqq 0 \quad \text { in } R \times[0, T] .
$$

Q.E.D.

Theorem 2.1 has now been proved combining Theorem 6.1 and Theorem 7.3. 


\section{REFERENCES}

[1] Bodanko, W.: Sur le problème de Cauchy et les problèmes de Fourier pour les équations paraboliques dans un domaine non borné, Ann. Polon. Math., 18 (1966), 79-94.

[2] Friedman, A.: Partial differential equations of parabolic type, Prentice-Hall (1964).

[ 3 ] Hörmander, L.: Pseudo-differential operators and hypoelliptic equations, Amer. Math. Soc. Symp. Pure Math. 10 (1966), Singular integral operators, 138-183.

[ 4 ] — : Fourier integral operators, I, Acta Math., 127 (1971), 79-783.

[5] — : Hypoelliptic second order differential equations, Acta Math., 119 (1968), $147-171$.

[6] Igari, K.: Degenerate parabolic differential equations, to appear.

[7] Kato, Y.: The hypoellipticity of degenerate parabolic differential operators, J. Functional Analysis, Vol. 7, No. 1 (1971), 116-131.

[8] Matsuzawa, T.: On some degenerate parabolic equations I, Nagoya Math. J. Vol. 51 (1973), 57-77.

[ 9 ] Mizohata, S.: Hypoellipticité des équations paraboliques, Bull. Soc. Math. France, 85 (1957), 15-50.

[10] Nirenberg, L. and Treves, F.: On local solvability of linear partial differential equations, Part I: Necessary conditions. Comm. Pure Appl. Math., Vol. 23 (1970), $1-38$.

[11] Oleinik, O. A.: On the smoothness of the solutions of degenerate elliptic and parabolic equations, Sov. Math. Dokl., 6 (1965), 972-976.

[12] _ - Linear equations of second order with non-negative form, Math. USSR-Sb., 69 (1966), 111-140 (in Russian). Amer. Math. Soc. Translation, 167-199.

[13] Treves, F.: A new method of proof of the subelliptic estimates, Comm. Pure Appl. Math., Vol. 24 (1971), 71-115.

[14] _-: Analytic-hypoelliptic partial differential equations of principal type, Comm. Pure Appl. Math., 24 (1971), 537-570.

[15] Schwartz, L.: Théorie des distributions, Vol. 1, Hermann, Paris (1957).

Department of Mathematics

Nagoya University 